\title{
XMM-Newton observation of the Seyfert 1.8 ESO 113-G010: Discovery of a highly redshifted iron line at $5.4 \mathrm{keV}$
}

\author{
D. Porquet ${ }^{1}$, J. N. Reeves ${ }^{2,3}$, P. Uttley ${ }^{2}$, and T. J. Turner ${ }^{2,4}$ \\ 1 Max-Planck-Institut für extraterrestrische Physik, Postfach 1312, 85741 Garching, Germany \\ e-mail: dporquet@mpe.mpg.de \\ 2 Laboratory for High Energy Astrophysics, NASA Goddard Space Flight Center, Greenbelt, MD 20771, USA \\ 3 Universities Space Research Association, 7501 Forbes Boulevard, Suite 206, Seabrook, MD 20706, USA \\ 4 Joint Center for Astrophysics, Physics Department, University of Maryland Baltimore County, 1000 Hilltop Circle, \\ Baltimore, MD 21250, USA
}

Received 2 June 2004 / Accepted 17 July 2004

\begin{abstract}
We present a spectral analysis of the Seyfert 1.8 ESO 113-G010 observed with XMM-Newton for 4 ks. The spectrum shows a soft excess below $0.7 \mathrm{keV}$ and more interestingly a narrow emission Gaussian line at $5.4 \mathrm{keV}$ (in its rest-frame), most probably originating from a redshifted iron $\mathrm{K} \alpha$ line. No significant line at or above $6.4 \mathrm{keV}$ is found contrary to other objects showing redshifted lines, ruling out a strong blue-wing to the line profile. The line is detected at $99 \%$ confidence, from performing Monte Carlo simulations which fully account for the range of energies where a narrow iron line is likely to occur. The energy of the line could indicate emission from relativistic $(0.17-0.23 \mathrm{c})$ ejected matter moving away from the observer, as proposed for Mrk 766 by Turner et al. (2004, ApJ, 603, 62). Alternatively, the emission from a narrow annulus at the surface of the accretion disk is unlikely due to the very small inclination angle (i.e. less than $10^{\circ}$ ) required to explain the narrow, redshifted line in this intermediate Seyfert galaxy. However emission from a small, localized hot-spot on the disk, occurring within a fraction of a complete disk orbit, could also explain the redshifted line. This scenario would be directly testable in a longer observation, as one would see significant variations in the energy and intensity of the line within an orbital timescale.
\end{abstract}

Key words. galaxies: active - galaxies: quasars: general - X-rays: general - line: formation -

X-rays: individual: ESO 113-G010

\section{Introduction}

In Active Galactic Nuclei (AGN), from Seyfert galaxies to quasars, the analysis of several X-ray features can help us to understand the central region of these powerful objects. Especially in the hard X-ray band, the $\mathrm{Fe} \mathrm{K} \alpha$ line complex observed in the 6-7 keV range is an important spectral diagnostic tool to probe dense matter from the inner disk out to the Broad Line Region and the molecular torus (see review in Reynolds \& Nowak 2003). Recently, narrow spectral features in the $5-6 \mathrm{keV}$ energy range were discovered with XMM-Newton and Chandra in a few AGN: NGC 3516 (Turner et al. 2002), ESO 198-G24 (Guainazzi 2003; Bianchi et al. 2004), NGC 7314 (Yaqoob et al. 2003), Mrk 766 (Turner et al. 2004), and IC 4329A (McKernan \& Yaqoob 2004). Localized spots or narrow annuli which occur on the surface of an accretion disk following its illumination by flares has been proposed to explain these features (e.g., Nayakshin \& Kazanas 2001; Turner et al. 2002; Dovčiak et al. 2004). An alternative scenario has been proposed by Turner et al. (2004) for Mrk 766 for which the first evidence for a significant line energy shift has been observed over a few tens of ks. They proposed that this shift could be interpreted as deceleration of an ejected blob of gas traveling close to the escape velocity.

Here, we present the first observation above $2 \mathrm{keV}$ by XMM-Newton of the Seyfert type 1.8 galaxy ESO 113-G010 $\left(z=0.0257, F W H M \mathrm{H} \alpha=2000 \mathrm{~km} \mathrm{~s}^{-1}\right.$, Pietsch et al. 1998). The present XMM-Newton data shows that this object shows a soft excess and more interestingly an emission feature near $5.4 \mathrm{keV}$ (in the Seyfert rest-frame). $H_{0}=75 \mathrm{~km} \mathrm{~s}^{-1} \mathrm{Mpc}^{-1}$ and $q_{0}=0.5$ are assumed throughout.

\section{XMM-Newton observation}

ESO 113-G010 was observed by XMM-Newton on May 3, 2001 (OBSID:0103861601). The EPIC MOS cameras (Turner et al. 2001) were operating in partial window mode (exposure time about $6.9 \mathrm{ks}$ ), and the EPIC PN camera (Strüder et al. 2001) was operating in full window mode (exposure time about $4.1 \mathrm{ks}$ ). The data were re-processed and cleaned using the XMM-Newton SAS VERSION 5.4.1 (Science Analysis Software) package. Data are selected using event patterns $0-4$ and $0-12$ for pn and MOS, respectively, whilst only good X-ray events (with "FLAG $=0$ ") are included. The source spectra are extracted from a circular source region of about $32.6^{\prime \prime}$ radius, 
and the background spectra are extracted from a box $\left(6^{\prime} \times 3^{\prime}\right)$ close to the source. The pn net exposure time is $4 \mathrm{ks}$. The $S / N$ of the MOS data is too low above $5 \mathrm{keV}$, therefore we use only the PN data which has the greatest sensitivity in the iron $K$-shell band. Unfortunately, for the present exposure time the $S / N$ of the RGS data are by far too low for any spectral analysis.

\section{Spectral analysis}

In the following analysis, abundances are set to those of Anders \& Grevesse (1989), whilst we use the updated crosssections for X-ray absorption by the interstellar medium from Wilms et al. (2000). The Galactic column density value is $2.74 \times 10^{20} \mathrm{~cm}^{-2}$, obtained from the COLDENS program using the compilations of Dickey \& Lockman (1990). We bin the background-subtracted spectrum in order to have a minimum of 20 counts per bin. The errors quoted correspond to $90 \%$ confidence ranges for one interesting parameter $\left(\Delta \chi^{2}=2.71\right)$.

First we fit the data in the $1-4 \mathrm{keV}$ energy range where the spectrum is relatively unaffected by the presence of a soft excess below about $0.7 \mathrm{keV}$, by a possible warm absorber, and by a Fe $\mathrm{K}$ emission line. Over this energy range, the data are well fitted by a single power law model with $\Gamma=2.07 \pm 0.08$ $\left(\chi^{2}=113.4 / 116\right)$. Figure 1 displays the spectrum extrapolated over the $0.3-8 \mathrm{keV}$ broad band energy. A strong positive residual is clearly seen below about $0.7 \mathrm{keV}$ due to the presence of a soft X-ray excess. In addition, a clear positive deviation near $5.4 \mathrm{keV}$ (in the Seyfert rest-frame) is observed.

\subsection{The soft $X$-ray spectrum}

The spectrum below $2 \mathrm{keV}$ is only moderately fitted $\left(\chi^{2} /\right.$ d.o.f. $\left.=268.5 / 197\right)$ by an absorbed (by Galactic absorption and intrinsic absorption) power law with $\Gamma=2.67_{-0.05}^{+0.08}$ (due mainly to the presence of a soft excess below $0.7 \mathrm{keV}$ ). We find an upper limit for intrinsic absorption at the redshift of the Seyfert of $0.8 \times 10^{20} \mathrm{~cm}^{-2}$. Since this value is much weaker than the Galactic absorption, we fix the absorption column density to that of the Galaxy (i.e., $2.74 \times 10^{20} \mathrm{~cm}^{-2}$ ). We inferred a $0.1-2.4 \mathrm{keV}$ luminosity of about $1.4 \times 10^{43} \mathrm{erg} \mathrm{s}^{-1}$, which is compatible with the previous observations with ROSAT (Pietsch et al. 1998). The standard explanation for the soft $\mathrm{X}$-ray excess is that it results from thermal emission originating directly from the hot inner accretion disk (Malkan \& Sargent 1982), and hence it is the high energy tail of the socalled Big Blue Bump. We fit the data with the combination of an accretion disk black body spectrum (DISKPN in XSPEC, Gierliński et al. 1999) and a power law continuum, for the soft and hard band, respectively. We find a rather good fit with $k T=121_{-10}^{+16} \mathrm{eV}$ and $\Gamma=1.94 \pm 0.07\left(\chi^{2} /\right.$ d.o.f. $\left.=267.9 / 250\right)$.

\subsection{The spectral feature at $5.4 \mathrm{keV}$}

Fitting the data above $1 \mathrm{keV}$ with a (Galactic) absorbed power law gives a good fit with $\Gamma=1.98 \pm 0.06\left(\chi^{2} /\right.$ d.o.f. $=$ $125.1 / 128)$. The inferred unabsorbed $2-10 \mathrm{keV}$ flux is $2.4 \times$ $10^{-12} \mathrm{erg} \mathrm{cm}^{-2} \mathrm{~s}^{-1}$, corresponding to a luminosity of $3.1 \times$ $10^{42} \mathrm{erg} \mathrm{s}^{-1}$. There is a clear positive residual near $5.4 \mathrm{keV}$ as

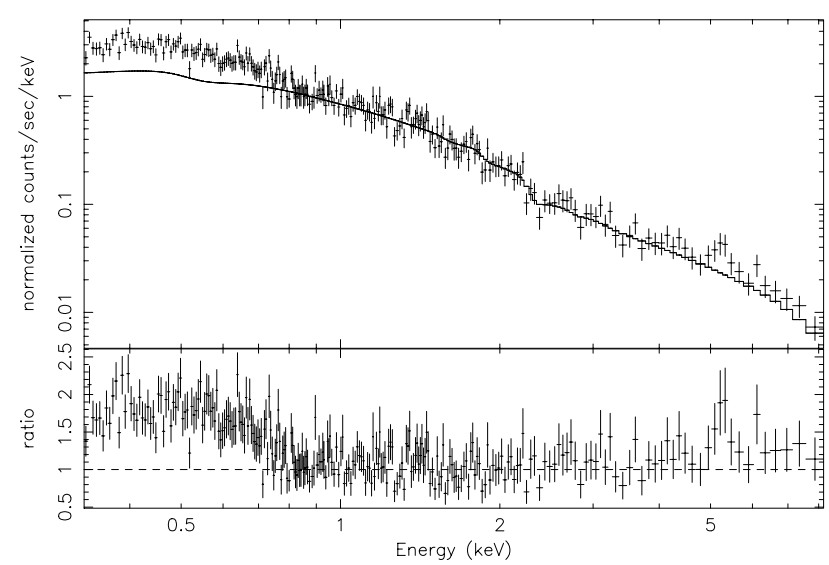

Fig. 1. PN spectrum of ESO 113-G010 $(z=0.0257$, observer frame). A power law has been fitted to the $1-4 \mathrm{keV}$ data and extrapolated to lower and higher energies. A soft X-ray excess is clearly seen extending to about $0.7 \mathrm{keV}$, as well as a positive deviation near $5.4 \mathrm{keV}$.

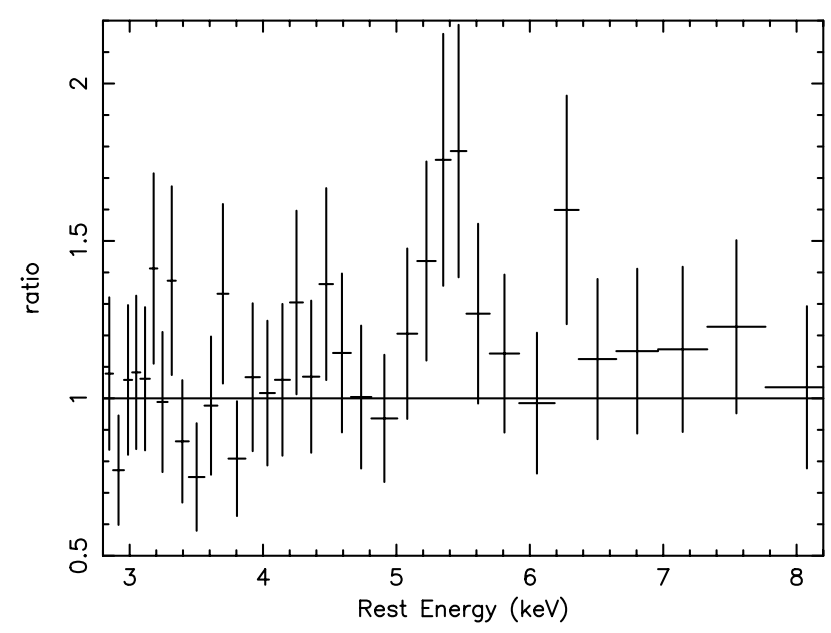

Fig. 2. A close-up of the data/model ratio in the $3-8 \mathrm{keV}$ energy range. The model is a Galactic absorbed power law. A clear positive residual is seen near $5.4 \mathrm{keV}$.

also shown in Fig. 2. The feature is well modeled by a Gaussian line (with $\sigma=0.1 \mathrm{keV}$ ) with $\Delta \chi^{2}=8.7$ for 2 additional parameters. The line energy is $5.38 \pm 0.11 \mathrm{keV}$ with $E W=265_{-145}^{+147} \mathrm{eV}$. Figure 3 shows the confidence contour plot for the rest-energy and intensity of the line corresponding to the $68 \%, 90 \%$ and 95\% confidence levels. One should notice that a $\mathrm{Cr} \mathrm{K} \alpha$ (at $5.4 \mathrm{keV}$ ) is present in the internal EPIC-pn background (see Fig. 2 in Freyberg et al. 2002) but its strength is negligible and therefore can be excluded as a possibility to explain the $5.4 \mathrm{keV}$ line. Formally, we do not find any statistical requirement for a narrow line at $6.4 \mathrm{keV}\left(\Delta \chi^{2}=0.8\right.$ for 1 additional parameter). Fixing the line energy at $6.4 \mathrm{keV}$ and the width at $10 \mathrm{eV}$ (i.e an intrinsically narrow line), we find an upper limit for the $E W$ of $230 \mathrm{eV}$. Splitting the observation into two equal parts (of about $2 \mathrm{ks}$ each), we do not find any significant line parameter variation during this very short timescale. 


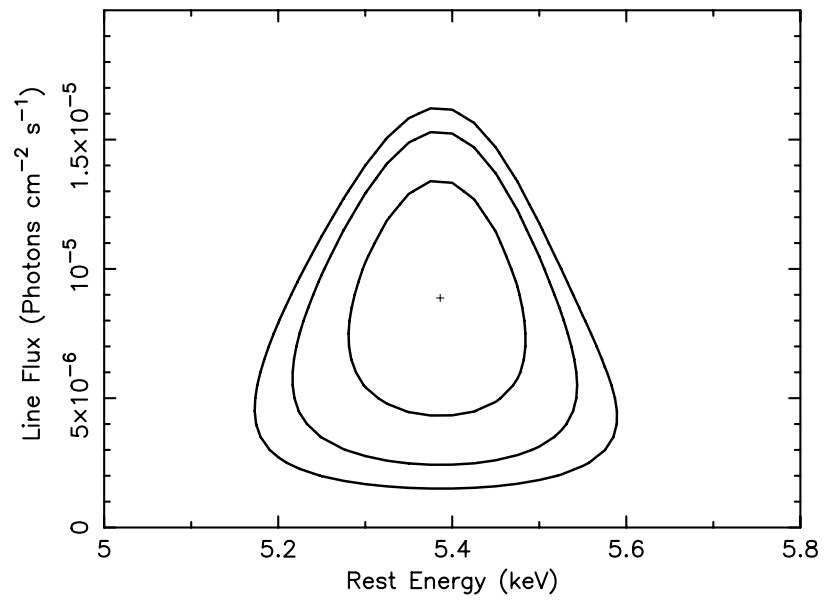

Fig. 3. Contour plot showing the $68 \%, 90 \%$ and $95 \%$ (from the inner to outer curves) confidence level for values of line rest-energy (keV) and line flux for the line detected near $5.4 \mathrm{keV}$.

\subsection{Estimating the detection probability of the line}

In estimating the significance of the $5.4 \mathrm{keV}$ line, straightforward application of the standard two parameter F-test does not take into account the range of energies where lines can occur in the spectrum, nor does it easily account for the number of bins in which one is searching for lines. The probability $P_{0}$ of finding a feature at one particular given energy can be estimated from the F-test, upon the addition of one extra degree of freedom (the line normalisation), which in ESO 113-G010 is $99.8 \%$. However in searching for redshifted (or blueshifted) iron lines, we are fitting over many bins or resolution elements, where narrow features may occur by chance (i.e. statistical noise). Thus the probability of detecting a line at any energy in the iron $K$-shell band can be estimated by $P_{N}=P_{0}^{N}$, where $N$ is the number of bins searched. In ESO 113-G010, we define the iron $K$ band between 4-7 keV (i.e. where one may realistically expect to find iron lines), which consists of 18 spectral bins (at 20 counts per bin). Thus the probability of detection of the $5.4 \mathrm{keV}$ feature by this estimate is $96.5 \%$. Note this is probably an under-estimate of the detection probability, as some of the actual spectral bins are smaller than the instrument resolution and therefore the number of bins (or trials) where a line may be located has probably been over-estimated.

We also carried out a more rigorous test of the significance of the line using Monte Carlo simulations. For our null hypothesis, we assumed that the spectrum is simply an absorbed power-law continuum, with the same parameters as the absorbed power-law model fitted to the real data. We used the XSPEC FAKEIT command to create 1000 fake EPIC-pn spectra corresponding to this model, with photon statistics expected from the $4 \mathrm{ks}$ exposure, and grouped each spectrum to a maximum of 20 counts per bin. Following the procedure used to test the real data for the presence of a narrow line, we fitted each fake spectrum with an absorbed power-law (absorption fixed at Galactic, but power-law photon index and normalisation left free to vary), to obtain a $\chi^{2}$ value. We then added a narrow line $(\sigma=0.1 \mathrm{keV})$ to the fit, restricting the line energy to be between 4-7 keV. Furthermore we stepped the line

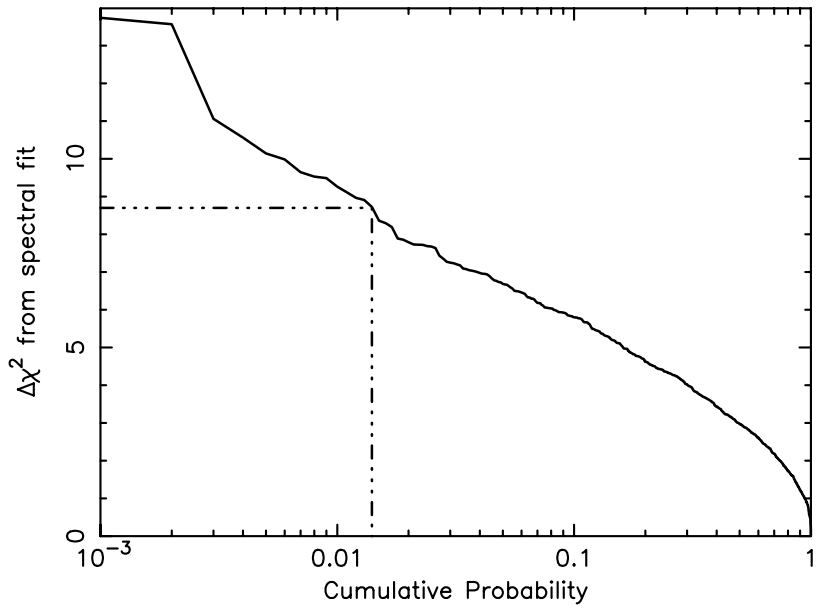

Fig. 4. The measured change in $\chi^{2}$ against cumulative probability for the addition of a single Gaussian line in 1000 randomly generated simulated spectra. The null hypothesis for the simulated spectra is a power-law continuum with Galactic absorption. For ESO 113-G010, the measured $\Delta \chi^{2}=8.7$ for the addition of a Gaussian line at $5.4 \mathrm{keV}$ is only exceeded in $1.4 \%$ of the simulated spectra. Therefore the $5.4 \mathrm{keV}$ line is detected at $\sim 99 \%$ confidence.

over the 4-7 keV energy range in increments of $0.1 \mathrm{keV}$, whilst fitting separately each time to ensure the lowest $\chi^{2}$ value was found. We then recorded the minimum $\chi^{2}$ obtained from these multiple line fits for each fake spectrum, and compared with the corresponding $\chi^{2}$ of the null hypothesis fits, to obtain 1000 simulated values of the $\Delta \chi^{2}$, which we used to construct a cumulative frequency distribution of the $\Delta \chi^{2}$ expected for a blind line search in the 4-7 keV range, assuming the null hypothesis of a simple power-law with no line is correct. The resulting distribution of $\chi^{2}$ against detection probability is shown in Fig. 4. We therefore find only $1.4 \%$ of fake power-law spectra fitted with a line show a larger $\Delta \chi^{2}$ than observed in the real data, implying that the line detection is significant at approximately $99 \%$ confidence.

\subsection{A relativistic disk origin for the line?}

The $5.4 \mathrm{keV}$ line could be a relativistic line for which we only see one horn due to the moderate spectral resolution. Therefore, we fit the data with a DISKLINE profile (Fabian et al. 1989). We assume a narrow annulus of $1 R_{\mathrm{g}}$, in order to obtain a narrow profile. Initially we fix the inclination angle at $\theta=45^{\circ}$, and assume the line is emitted at $6.4 \mathrm{keV}$ (i.e near-neutral iron). However this model fails to re-produce the narrow emission line at $5.4 \mathrm{keV}$. Upon varying the disk inclination angle a good fit to the line is obtained, with $\chi^{2} /$ d.o.f. $=118.4 / 127$, requiring a very small inclination angle of $\theta \sim 5^{\circ}$, with a formal upperlimit at $90 \%$ confidence of $\theta<10^{\circ}$. We constrain the inner radius of the annulus at $R_{\text {in }}=9.5_{-0.9}^{+1.1} R_{\mathrm{g}}$, whilst the line $E W$ is $303 \pm 166 \mathrm{eV}$. Thus for a very low (face-on) disk inclination the line is gravitationally redshifted whilst there is very little transverse velocity shift, hence the red and blue wings of the line are not resolved. A narrow redshifted feature is then observed, consistent with the line profile in Fig. 2. At higher disk inclination angles, the transverse velocity shift is much more 

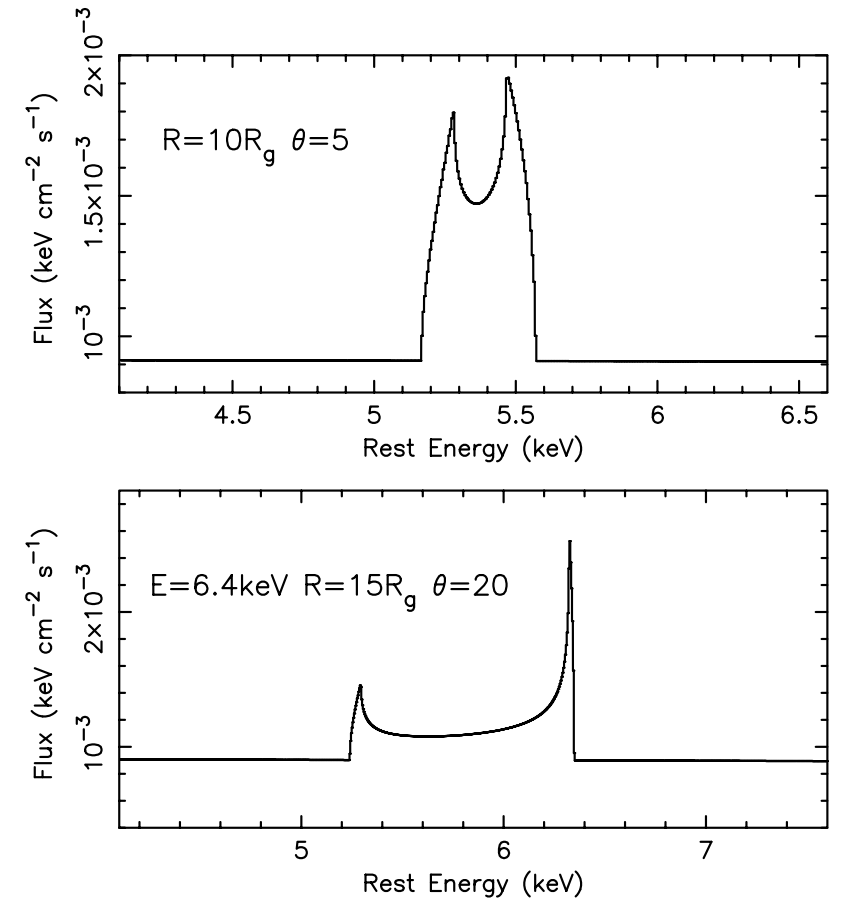

Fig. 5. Line profiles obtained by a relativistic line model for two inclination angles $(\theta)$. Top: $\theta=5^{\circ}$. Bottom: $\theta=20^{\circ}$.

prominent leading to a strong blue wing, which is not observed in the data. This is illustrated in Fig. 5 where the theoretical line profiles at two angle inclinations are compared, i.e., $\theta=5^{\circ}$ and $\theta=20^{\circ}$.

\section{Discussion}

It has been proposed by Skibo (1997) that energetic protons can be responsible of the destruction (spallation) of $\mathrm{Fe}$ into $\mathrm{Cr}$ and other lower $Z$ metals on the surface of the accretion disk, which enhances the line emission expected from elements of low abundance. The strongest line apart the $\mathrm{Fe} \mathrm{K} \alpha$ line expected is the $\mathrm{Cr} \mathrm{K} \alpha$ line at $5.4 \mathrm{keV}$, and the second one being the $\mathrm{Mn} \mathrm{K} \alpha$ at $5.9 \mathrm{keV}$ (see Fig. 4 in Skibo 1997). The energy of the feature we find here is compatible with the energy of the $\mathrm{Cr} \mathrm{K} \alpha$ line. However the ratio of the line at $5.4 \mathrm{keV}$ (if $\mathrm{Cr} \mathrm{K} \alpha$ ) and the upper limit of a narrow Gaussian at $6.4 \mathrm{keV}$ (due to iron) is not consistent with the line ratio predicted by this model, i.e. the line flux of the $\mathrm{Cr} \mathrm{K} \alpha$ should represent $33 \%$ of the Fe K $\alpha$. This is not compatible with $E W=265_{-145}^{+147} \mathrm{eV}$, and $E W<230 \mathrm{eV}$ found respectively at $5.4 \mathrm{keV}$ and $6.4 \mathrm{keV}$. Therefore iron spallation seems to be an unlikely explanation in this case, as also found for other similar line observations (e.g., NGC 3516 and Mkn 766).

If instead the line originates from an annulus on the disk, then the small inclination angle derived above does not appear to be consistent with the classification of this object as a type 1.8 Seyfert, as one might expect a larger inclination angle of about $45-60^{\circ}$. However, Nayakshin \& Kazanas (2001), Turner et al. (2002) for NGC 3516, and Dovčiak et al. (2004) have proposed that these features could be due to localized hotspots on the accretion disk surface following its illumination by flares.
In this case, the range of transverse velocity shifts would be much smaller than for an entire disk orbit, resulting in a relatively narrow emission line feature, even at lower inclination angles. Narrow red-shifted features could be produced with an inclination angle of up to about 30 degrees in this model (see Fig. 1 in Nayakshin \& Kasanas 2001, and also Fig. 3 in Dovčiak et al. 2004), while at higher inclination angles the line becomes blue-shifted above $6.4 \mathrm{keV}$, which is ruled out from the data. The narrow width of the observed emission line would imply that the emitting region must be small and that it is observed for only a fraction of the entire orbit. Indeed the orbital timescale of matter co-rotating along a circular trajectory assuming $r=10 R_{\mathrm{g}}$ is between $2.7-27 \mathrm{~h}$ for a black mass of $10^{7} M_{\odot}$ and $10^{8} M_{\odot}$, respectively, probably larger than the exposure time of the present observation. A longer exposure is therefore required to study possible variations in the line profile as a function of phase of the spot.

The redshifted line emission could be also due to the signature of an inflow or outflow of matter from material close to the nucleus. Moving material that is not part of the disk structure would be sensitive to the effects of strong gravity and may achieve velocities that are a significant fraction of the speed of light, causing significant displacement of emission lines from their rest energy. In the XMM-Newton spectrum of Mrk 766, Turner et al. (2004) found an energy shift from 5.60 to $5.75 \mathrm{keV}$ in a line occurring over a few tens of ks. They proposed that such rapid energy shift could be interpreted as deceleration of an outflowing ejected blob of gas traveling to the escape velocity, where the material is viewed on the reverse side of the black hole (i.e. moving away from the observer). The energy of the line in ESO 113-G010 could indicate a (redshifted) fast moving medium at $0.16-0.23 \mathrm{c}$, assuming a neutral iron $(6.4 \mathrm{keV}$ in its rest-frame) or a $\mathrm{H}$-like iron ( $7 \mathrm{keV}$ in its rest-frame) respectively.

Up to now mainly red-shifted narrow lines (except for the NLS1 RX J0136.9-3510, Ghosh et al. 2004) have been observed in Seyfert 1 galaxies. For the hotspot model, this could be explained by the fact that red-shifted lines are predicted for very small disk inclination (up to about 30 degree), which is compatible with the Unified Scheme (Antonucci 1993). For the blob model, this could be explained also by a geometry effect. The outflow could be optically thick on the near side of the flow, so one only sees emission from the reverse side. However given the statistical quality of this dataset at energies above the iron $K$ line $(>7 \mathrm{keV})$, it is not possible to exclude a blue-shifted component to the line from an outflow, which has the same strength as the redshifted $5.4 \mathrm{keV}$ line. For instance the $90 \%$ confidence upper limit on the equivalent width of a line detected between $7-8 \mathrm{keV}$ is $<340 \mathrm{eV}$, i.e. consistent with the line observed at $5.4 \mathrm{keV}$.

The present observation of ESO 113-G010 is far too short (4 ks) to study any shift of the line energy and measure any outflow or inflow of the material. Indeed within the current snapshot observation, three scenarios may be possible; (i) the line emitting matter may be accelerating due to gravity onto the accretion disk or black hole; (ii) the material may be ejected from the accretion disk and decelerate under gravity away from the observer or (iii) the line may originate from a rapidly variable 
hot-spot on the disk surface. In case of a material accelerating or decelerating under gravity, we can use $\Delta v / \Delta t=G M / R^{2}$ (where $\Delta t$ is the time under which the deceleration is observed and $R$ is the radius at which the material is situated at that time) to estimate the observed time of velocity change. For example, assuming a change of the energy line from $5.4 \mathrm{keV}$ to $5.6 \mathrm{keV}$, and $R \sim 3.5 \times 10^{14} \mathrm{~cm}$ (as for Mrk 766, Turner et al. 2004), we inferred $\Delta t \sim 90 \mathrm{ks}$ and $\Delta t \sim 9 \mathrm{ks}$, assuming a black hole mass of $10^{7} M_{\odot}$ and $10^{8} M_{\odot}$, respectively. In the case of the hotspot scenario, both line energy and line intensity are predicted to vary with time on the orbital timescale (from $10 \mathrm{ks}$ up to $100 \mathrm{ks}$ if the black hole mass is $10^{7} M_{\odot}$ and $10^{8} M_{\odot}$, respectively), as shown in Fig. 1 in Nayakshin \& Kazanas (2001) (see also Figs. 3 and 4 in Dovčiak et al. 2004). Therefore a much longer XMM-Newton observation, of about $100 \mathrm{ks}$ in length, is required to be able to differentiate between these models.

In this paper we have performed Monte Carlo simulations to calculate a realistic detection probability for the redshifted line at $5.4 \mathrm{keV}$. The Monte Carlo method takes into account that an iron line-like residual may be detected at any energy within the iron line band (defined as $4-7 \mathrm{keV}$ in this instance); in contrast the conventional F-test is likely to over-estimate the detection significance, as this does not account for the range of energies or bins where a redshifted line may be observed. Applying the Monte-Carlo method to ESO 113-G010 results in a detection significance of $99 \%$, which is still just under the $3 \sigma$ confidence level. Clearly, a longer observation will be needed to increase the detection significance of the feature to $>99 \%$ confidence. This is also the first time a Monte-Carlo probability estimate has been reported for one of the several previously published narrow redshifted line features in AGN, e.g., NGC 3516 (Turner et al. 2002), ESO 198-G24 (Guainazzi 2003; Bianchi et al. 2004), NGC 7314 (Yaqoob et al. 2003), Mrk 766 (Turner et al. 2004), and IC 4329A (McKernan \& Yaqoob 2004). It would therefore be desirable to re-evaluate earlier claims on redshifted line detections using more robust Monte-Carlo techniques, as well as analysing a wider sample of Seyfert galaxies to determine the frequency of the relativistically shifted lines in AGN.
Acknowledgements. Based on observations obtained with the XMM-Newton, an ESA science mission with instruments and contributions directly funded by ESA member states and the USA (NASA). The authors thank the anonymous referee for fruitful comments and suggestions. D.P. acknowledges grant support from an MPE fellowship.

\section{References}

Anders, E., \& Grevesse, N. 1989, Geochim. Cosmochim. Acta, 53, 197

Antonucci, R. 1993, ARA\&A, 31, 473

Bianchi, S., Matt, G., Balestra, I., Guainazzi, M., \& Perola, G. C. 2004, A\&A, 422, 65

Dickey, J. M., \& Lockman, F. J. 1990, ARA\&A, 28, 215

Dovčiak, M., Bianchi, S., Guainazzi, M., Karas, V., \& Matt, G. 2004, MNRAS, 350, 745

Fabian, A. C., Rees, M. J., Stella, L., \& White, N. E. 1989, MNRAS, 238,729

Freyberg, M., Pfeffermann, E., \& Briel, U. 2002, in New Visions of the X-ray Universe in the XMM-Newton and Chandra Era, ed. F. Jansen (The Netherlands: ESTEC), in press [arXiv: astro-ph/06406]

Ghosh, K. K., Swartz, D. A., Tennant, A. F., et al. 2004, ApJ, 607, L111

Gierliński, M., Zdziarski, A. A., Poutanen, J., et al. 1999, MNRAS, 309, 496

Guainazzi, M. 2003, A\&A, 401, 903

Malkan, M. A., \& Sargent, W. L. W. 1982, ApJ, 254, 22

McKernan, B., \& Yaqoob, T. 2004, ApJ, 608, 157

Nayakshin, S., \& Kazanas, D. 2001, ApJ, 553, L141

Pietsch, W., Bischoff, K., Boller, Th., et al. 1998, A\&A, 333, 48

Porquet, D., Kaastra, J. S., Page, K. L., et al. 2004, A\&A, 413, 913

Reynolds, C. S., \& Nowak, M. A. 2003, Phys. Rep., 377, 389

Skibo, J. G. 1997, ApJ, 478, 522

Strüder, L., Briel, U., Dennerl, K., et al. 2001, A\&A, 365, L5

Turner, M. J. L., Abbey, A., Arnaud, M., et al. 2001, A\&A, 365, L27

Turner, T. J., Mushotzky, R. F., Yaqoob, T., et al. 2002, ApJ, 574, L123

Turner, T. J., Kraemer, S. B., \& Reeves, J. N. 2004, ApJ, 603, 62

Wilms, J., Allen, A., \& McCray, R. 2000, ApJ, 542, 914

Yaqoob, T., George, I. M., \& Kallman, T. R., et al. 2003, ApJ, 596,85 